\title{
Detection of genetic factors controlling pollen-style interaction in maize
}

\author{
MIRELLA SARI-GORLA*, GIORGIO BINELLI, M. ENRICO PÉ \& MARZIO VILLA \\ Department of Genetics and Microbiology, University of Milan, Via Celoria 26, 20133 Milan, Italy
}

\begin{abstract}
In order to detect loci specifically related to pollen-style interaction in maize, linkage analysis to RFLP markers was performed on a population of recombinant inbred lines, the pollen of which was used to pollinate female plants of two different genotypes: the $F_{1}$ from which the recombinant inbred population was derived ('self female') and a genetically unrelated hybrid genotype ('cross female'). Besides pollen grain germinability and pollen tube growth rate on the two females, pollen general competitive ability and pollen specific competitive ability for both traits were evaluated, following the procedure used to estimate general and specific combining ability, applied to the pollen-style genetic combination instead of to the hybrid genetic combination. A clear advantage of recombinant inbred line pollen, in terms of germination and early growth, was revealed on self female tissues whereas an inverse effect was detected for tube growth rate: tube growth, until fertilization, was faster on the female of unrelated genotype. Linkage analysis to molecular markers (RFLP) of germinability and of tube growth rate on the two stylar genotypes identified nine QTLs for the first trait on the self and eight on the cross female; four chromosome regions for tube growth rate were detected on the self and 11 on the cross style. Most of the general competitive ability effects were localized in the regions where QTLs for germinability or pollen tube growth were detected, in particular on the cross female; on the contrary, most of the loci for pollen specific competitive ability effects proved to be localized in different chromosome regions.
\end{abstract}

Keywords: competitive ability, maize, pollen, pollen-style interaction, QTLs, RFLP analysis.

\section{Introduction}

The reproductive success of a pollen grain, its fertilization ability, is the result of many adaptive characteristics: pollen genotypes having efficient microspore development, and hence good viability, high percentage and speed of germination and high tube growth rate, will compete successfully against other pollen grains to fertilize the ovule (Ottaviano \& Mulcahy, 1989).

The postshedding components of pollen competitive ability, pollen germination and tube growth, are very sensitive to, and dependent on, the environment (Brewbaker \& Kwack, 1963; Jennings \& Topham, 1971). Germination ability is controlled, even in compatible species, by a complex system of signalling, precluding hydrating and tube extrusion until grains have landed on a stigma (Dickinson, 1993). Pollen tube growth is relatively independent of stylar nutrients only during the first phases of growth; subsequently, pollen function can be strongly affected by the female tissues

\footnotetext{
${ }^{*}$ Correspondence.
}

in which it grows, and that can act as a sieve, favouring the more competitive gametophytes. In maize, the establishment of a metabolic interaction between pollen and style is expected for physiological reasons: because of the difference between pollen diameter $(90-100 \mu \mathrm{m})$ and tube length $(20 \mathrm{~cm}$ or more) (Heslop-Harrison, 1987; Ottaviano \& Mulcahy, 1989; Sari-Gorla \& Frova, 1994).

In some cases the biological bases of these effects has been ascertained; in Leucaena leucocephala stigmatic inhibition of pollen grain germination when the pollen grains are less than a critical number in the stigma is due to a $\mathrm{pH}$-dependent proteinaceous inhibitor, active at the stigmatic $\mathrm{pH}$ (Ganeshaiah \& Uma Shaanker, 1988). In peach, pollen tube growth has been related to changes in the pistil structures which the pollen tube has to pass through on its way from the stigma to the ovule: starch, present all along the stylar tract at anthesis, vanishes as the pollen tubes pass by, indicating that the pollen tube growth is heterotrophic (Herrero \& Arbeloa, 1989).

The genetic control of the pollen-pistil interaction is fundamental in the determination of the reproductive system of the plants: it can produce the extreme 
situation of exclusive cross-fertilization as well the opposite condition of reproductive isolation. These cases, the first in particular, have been extensively investigated (see Clarke \& Newbigin, 1993, for review), whereas information concerning the phenomenon in self-compatible species is scarce. The genetic basis of pollen-style interaction has been demonstrated in different species (Gawel \& Robacker, 1986; Pedersen, 1988) and in maize (Pfahler, 1967; SariGorla et al., 1976; Ottaviano et al. 1980, 1983), but few specific genes directly involved in its control have been identified.

In vivo pollen growth exhibits quantitative variability (Sari-Gorla et al., 1992); thus the approach we adopted to detect loci specifically involved in the control of pollen-style interaction in maize was based on the use of molecular markers to analyse pollen function in different biological environments. RFLP analysis for pollen competitive ability was performed on a population of recombinant inbred (RI) lines; pollen from each RI line was used to pollinate female plants of two different genotypes: the $F_{1}$ from which the RI population was derived and female plants genetically unrelated to the pollen source.

\section{Materials and methods}

Linkage analysis to RFLP markers of pollen traits was based on a set of 44 recombinant inbred lines (RI), kindly supplied by B. Burr, obtained from inbreds T232 and CM37 and typed for 200 RFLP loci (Burr et al., 1988).

\section{Crossing design and pollen trait evaluation}

Pollen of each RI line was used to pollinate two different hybrid genotypes: the first was the $F_{1}$ between the parents T232 and CM37, henceforth indicated as 'self female', and the second was a A632 $\times$ Mo17 hybrid, genetically unrelated to the pollen source and indicated as 'cross female'. Five pollinations for each RI line were made on each female on the same day, and the experiment was repeated for 2 days. Thus, 10 ears per female and per pollen source were produced.

Two pollen traits were evaluated: pollen germinability and early growth, and late pollen tube growth rate, up to fertilization. The pollen characters were measured in vivo by means of a mixed pollination technique: equal amounts of the pollen to be tested and the pollen of a standard genotype (W22), carrying a genetic marker for coloured aleurone, were mixed and used to pollinate a female plant. Because the style length in maize varies according to the position of the flowers on the ear, increasing from the apex to the base, relative pollen tube growth rate (PTGR) can be expressed as the increase of the proportion of uncoloured kernels on the resulting ear: each ear was divided into four or five segments eight kernels long, according to their length, on which the proportion of uncoloured kernels was computed. The proportion of uncoloured kernels in the apical segment of the ear gives a measure of pollen germinability (in terms of time and rate) and of early tube growth, which will be referred to as pollen grain germination (PGG).

\section{Analysis of trait variability}

Analysis of variance (ANOVA) was performed on both PGG and PTGR. Because the data produced are binomial proportions and the number of observations for each of them, although very high (about 500), was not constant, the vector of observed proportions was linearly transformed by means of an operator matrix, in which rows correspond to the orthogonal polynomial coefficients that define the linear component (Ottaviano et al., 1988). The variables generated in this way allowed the evaluation of PGG as expected values in the first segment, before transformation, and PTGR as the regression coefficient of the uncoloured kernel proportion on the ear segments, after the transformation. Both of these variables were submitted by SAS GLM procedure (SAS, 1985) to a weighted least squares analysis, the weights being the reciprocals of the proper variances.

\section{Evaluation of pollen general and specific competitive ability}

The phenotypic values for both traits were partitioned into general and specific competitive ability (pollen general competitive ability, PGCA, and pollen specific competitive ability, PSCA): the procedure was that used to estimate general combining ability and specific combining ability, but in this case, instead of hybrid genetic combinations, pollen-style genetic combinations were considered (Ottaviano et al., 1980). Accordingly, PGCA, the mean performance of a genotype, was expressed as the deviation from the overall mean of the RI lines, and PSCA was computed as the deviation of a specific pollen-style combination from the mean values of the male and the female genotypes.

\section{QTL analysis}

The detection of the chromosome segments carrying loci affecting the trait components' variability was carried out on eight characters: PGG on the two females, PTGR in the two stylar tissues, and PGCA and PSCA for both the traits. 
The association between the expression of each trait and the molecular markers was detected by a one-at-atime marker analysis, comparing the marker class phenotypic means by: (i) one-way weighted analysis of variance, and (ii) weighted linear regression. The dependent variables were: PGG and PTGR mean predictions, classified by male and by female genotype, obtained on the basis of the ANOVA model; PGCA and PSCA effects for each character. For the ANOVA, the classification of genotypes was 1,2 (homozygous for CM37 or T232 alleles) or 1.5 (heterozygous locus). The weights were calculated from standard errors. In this analysis two or three levels of classifying factor were present; for the cases with three groups, the sum of squares for main effects was subdivided into two components: linear effects and quadratic effects. For the analysis of regression, the allelic composition of the molecular marker was taken as regressor, assuming the value of 1,2 or 1.5 as defined for ANOVA. A significant effect in ANOVA or a significant regression is interpreted as an indication that a quantitative trait locus (QTL) is closely linked to that RFLP locus.

All the computations were made by SAS GLM procedure.

In order to avoid false assignments, for each trait component the correlation matrices between the allelic values of all the restriction loci giving a significant result were computed, and only uncorrelated markers were retained. In fact, a cluster of significant markers does not necessarily indicate the presence of a corre-

(a)

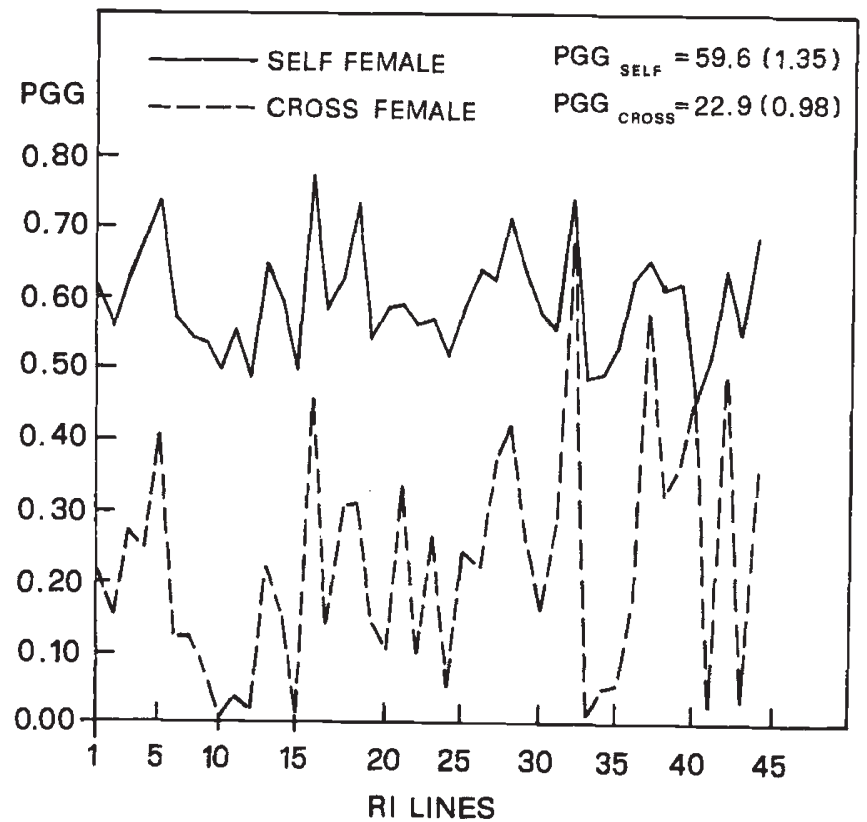

sponding number of QTLs, and may be due to linkage between one QTL and many flanking markers. Moreover, loci on different chromosomes may be correlated as a result of random drift occurring during the generations of inbreeding involved in the RI line construction. The cumulative contribution of the identified QTLs to the character variability was evaluated by multiple regression, including in the model all the significant, uncorrelated loci.

The statistical approach adopted, consisting of a 'guided' stepwise regression, was chosen because a multiple regression procedure might yield results not easily explained in terms of a genetic model (Dudley, 1993).

\section{Results}

\section{Pollen-style interaction}

The ANOVA results, not reported here for the sake of brevity, confirmed the high variability of PGG and PTGR in the RI lines (Sari-Gorla et al. 1992), and indicated a highly significant difference in their performance on the two females with regard to both traits.

In particular (Fig. 1), PGG was much higher on the self than on the cross female. As the trait was evaluated in relation to a tester line, the expected value, in the absence of a competitive advantage of one pollen type over the other, is around 50 per cent; however, this

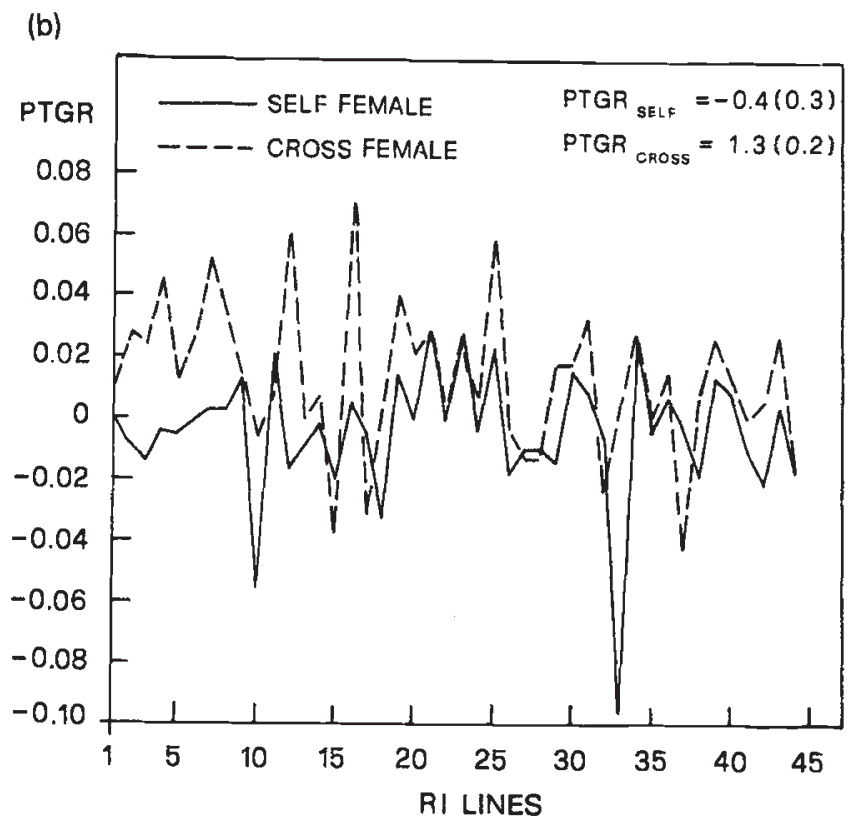

Fig. 1 Single RI lines performance for PGG (left) and PTGR (right) on self and cross females. Mean values (SE) of the traits are indicated. 
proportion was higher than expected on the self female and lower on the cross female. Opposite results were obtained for PTGR: its mean value was negative, meaning that the competitive ability of the tested pollen was lower than that of the standard line in the self combination, but was positive on the cross stylar tissues, where the pollen of RI lines was more competitive than the standard. It appears that RI pollen is very well adapted for germination and early growth on styles of similar genotype whereas, during the later stages of its function, it is more competitive on stylar tissues of unrelated genotype, indicating that a positive interaction is established between pollen and pistil of different genetic origins.

Correlation (not shown) between PGG on self and on cross females was significant and positive, as also between PTGR in the two stylar environments (Fig. 1). This indicates that the advantage in PGG in self combination and the disadvantage in PTGR in cross combination are similar for all the RI line set, and that the differences between line performances are maintained in both situations. This was confirmed by the correlation between PGG and PTGR values within females, which was significant and negative, indicating that the lines performing better during early growth are less competitive in the later stages of pollen function, and vice versa.

\section{Marker-associated QTLS}

The study of association between RFLP loci and QTLs controlling the traits was carried out by ANOVA and regression analysis. The sums of squares for regression are the same as for linear effects in ANOVA, but the $F$ values are different because in ANOVA quadratic effects are also taken out of the sums of squares. The analysis of regression reveals as significant only those loci for which the means for different genotypes are distributed linearly; for the same loci, ANOVA shows significant main effects and significant linear effects. In
Fig. 2 RFLP analysis of pollen grain germinability (PGG) evaluated on self female $(*)$ and cross female $(\bullet)$, of PGG general competitive ability $(\boldsymbol{\Delta})$ and specific competitive ability ( $)$. Horizontal bars indicate the degree of correlation between significant marker loci and the expression of the trait. In the case of loci significant for more than one trait, the bar is relative to the highest $\mathbf{R}^{2}$. The numbers refer to chromosomes. See text for more detailed explanations.

Fig. 3 RFLP analysis of pollen tube growth rate (PTGR) evaluated on self female $(*)$ and cross female $(\bullet)$, of PTGR general competitive ability $(\Delta)$ and specific competitive ability ( $)$. Horizontal bars indicate the degree of correlation between significant marker loci and the expression of the trait. In the case of loci significant for more than one trait, the bar is relative to the highest $\mathbf{R}^{2}$. The numbers refer to chromosomes. See text for more detailed explanations.

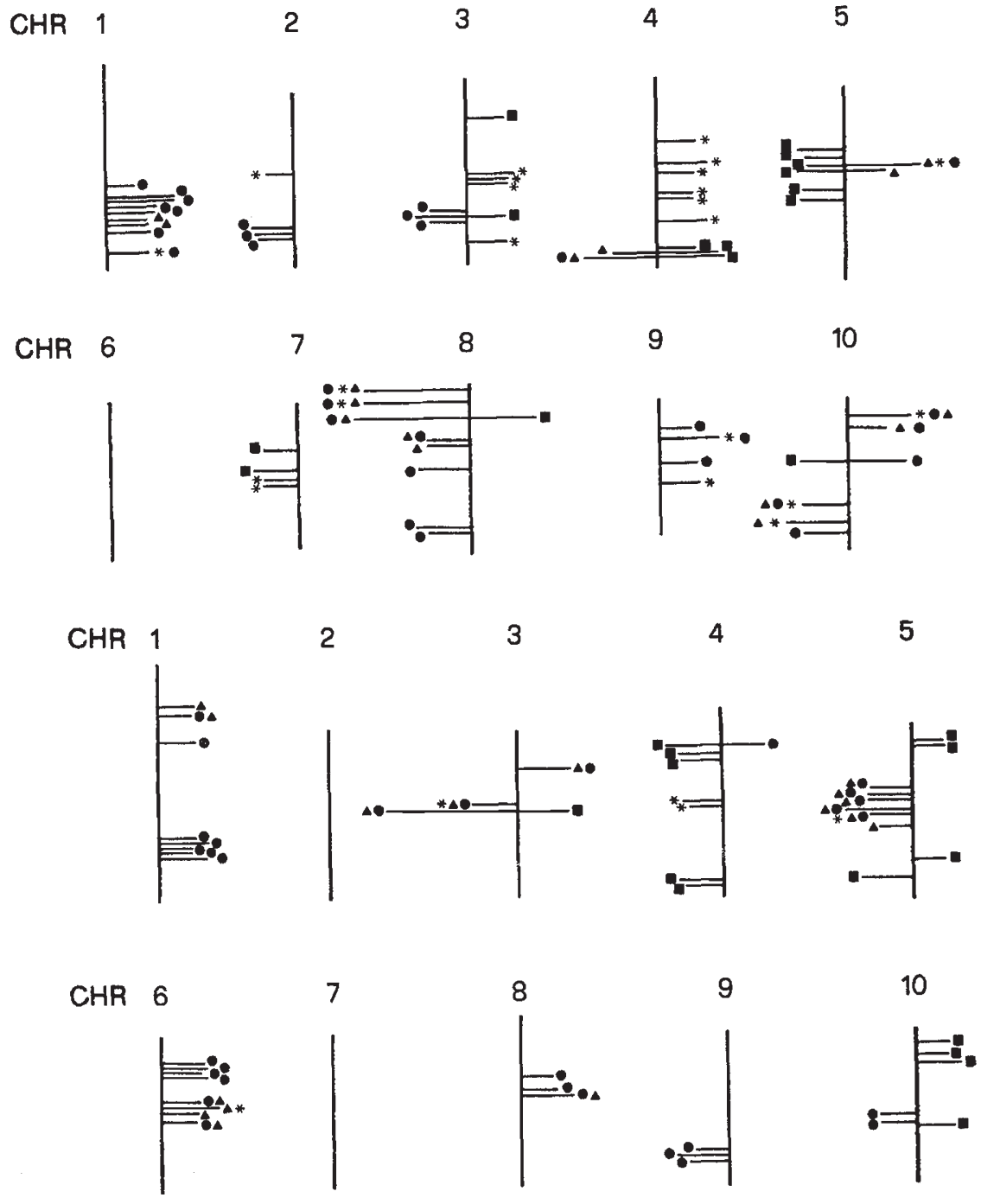


addition, ANOVA detects the loci with nonadditive effects because of the significant quadratic effects, thus providing more information than regression. However, we chose to perform a well-protected analysis, considering as important only those loci revealing both a significant linear effect in ANOVA and a significant regression.

Figures 2 and 3 report the chromosomal localization of QTLs involved in PGG and PTGR determination, respectively, detected by RFLP analysis on the basis of their significant association with the molecular markers, on the basis of the restrictions described above. The bars indicate the coefficient of determination, i.e. the proportion of the phenotypic variability of the trait explained by allelic substitution at the individual molecular marker; a bar to the left indicates a negative coefficient of regression, a bar to the right a positive one. In the first case, the allele increasing the trait is carried by the parental line CM37, in the second by the T232 line. As more than one marker will appear to be significantly associated with only one QTL, because of linkage, the putative presence of only one QTL is inferred for each cluster of significant markers; moreover, only loci not correlated with markers on other chromosomes were considered.

In classical quantitative genetics, inbred lines are evaluated according to how they perform in different hybrid combinations. The mean performance of a line is indicative of its general combining ability, the deviation of performance of a particular cross from the average of the two parental lines is the specific combining ability of that cross (Falconer, 1989). Pollen tubes within a style, like the parental genome in an $F_{1}$ hybrid, represent an interacting combination which was analysed, according to the concepts of general and specific competitive ability, with regard to both PGG and PTGR.
The analysis of association between PGG expression and the allelic composition at the restriction loci revealed 21 significant loci on the self female and 30 on the cross (Fig. 2 and Table 1); respective $R^{2}$ values ranged from 0.09 to 0.35 . On the basis of the criteria adopted in this analysis, nine chromosomal regions where factors involved in the trait determination are located were detected on the self female; in most cases the parent bearing the allele increasing the trait was CM37. On the cross female eight regions were detected.

Fifteen molecular markers were significantly associated with PGCA and 15 with PSCA effects related to PGG, the $\mathrm{R}^{2}$ values of which ranged from 0.10 to 0.25 . Five regions were detected where pollen GCA effects are located; all of them coincide with regions where QTLs for PGG were detected in the cross female. Instead, most of the PSCA loci were located in positions not associated with other QTLs.

In Fig. 3 the chromosomal location of QTLs involved in PTGR determination is indicated. The density of significant loci detected revealed a noticeable difference whether pollen grew into self stylar tissues or in cross combination (Table 1); five RFLP loci were significant on the self female and 30 on the cross female, allowing the identification of four regions in the first case and 11 in the second. The corresponding $R^{2}$ values ranged between $0.10-0.13$ and $0.10-0.39$, respectively.

Sixteen significant associations were observed between molecular markers and PGCA and four between markers and PSCA. Of the six regions revealing the presence of PGCA effects, five were the same detected for PTGR in the cross female. One region was common to all the trait components, two QTLs for PSCA effects were expressed on the cross female also, but four were exclusively for specific effects.

Table 1 Number of significant loci detected by RFLP analysis, range of respective $R^{2}$ values, number of putative QTLs (uncorrelated loci) and total $\mathrm{R}^{2}$, including only uncorrelated loci, for PGG and PTGR measured on self and on cross females, pollen general competitive ability (PGCA) and pollen specific competitive ability (PSCA) of the same traits

\begin{tabular}{|c|c|c|c|c|c|c|c|c|}
\hline & \multicolumn{4}{|c|}{ PGG } & \multicolumn{4}{|c|}{ PTGR } \\
\hline & $\begin{array}{l}\text { Self } \\
\text { female }\end{array}$ & $\begin{array}{l}\text { Cross } \\
\text { female }\end{array}$ & PGCA & PSCA & $\begin{array}{l}\text { Self } \\
\text { female }\end{array}$ & $\begin{array}{l}\text { Cross } \\
\text { female }\end{array}$ & PGCA & PSCA \\
\hline $\begin{array}{l}\text { No. of significant } \\
\text { loci }\end{array}$ & 22 & 30 & 15 & 15 & 5 & 30 & 16 & 14 \\
\hline Range of $R^{2}$ values & $\begin{array}{l}0.09 \\
0.22\end{array}$ & $\begin{array}{l}0.09 \\
0.35\end{array}$ & $\begin{array}{l}0.11 \\
0.25\end{array}$ & $\begin{array}{l}0.10 \\
0.19\end{array}$ & $\begin{array}{l}0.10 \\
0.13\end{array}$ & $\begin{array}{l}0.10 \\
0.39\end{array}$ & $\begin{array}{l}0.10 \\
0.26\end{array}$ & $\begin{array}{l}0.10 \\
0.15\end{array}$ \\
\hline No. of QTLs & 9 & 8 & 5 & 7 & 4 & 11 & 6 & 7 \\
\hline Total $\mathrm{R}^{2}$ & 0.64 & 0.39 & 0.47 & 0.61 & 0.30 & 0.73 & 0.63 & 0.52 \\
\hline
\end{tabular}


Table 1 reports the values of total $\mathrm{R}^{2}$ for each trait: this measures the amount of phenotypical variability explained by QTLs linked to the marker loci included in the model. The values were rather high, except for PGG evaluated on the cross female and PTGR on the self female, indicating that most of the QTLs involved in the trait control and segregating in the population had been detected.

\section{Discussion}

A clear advantage of the pollen from RI lines, in terms of germination and early growth, was revealed on self female tissues whereas an inverse effect was detected for tube growth rate: subsequent tube growth, up to fertilization, was better on the female of unrelated genotype. It has to be borne in mind, however, that all the data have been obtained relative to a standard line, W22. Thus, when considering pollen performance of the RI lines relative to other tester lines, different QTLs and different behaviours of self and cross pollen could be observed.

In general, the data so far published with regard to the genetic control of the pollen-style interaction are in favour of a tendency toward assortative mating. The results of an experiment designed to test the effect of pollen-style interaction in maize (Ottaviano et al., 1983) by comparing the pollen competitive ability of self pollen with that of cross pollen, indicated that when divergent genotypes are competing in the same style, the interaction with the maternal tissues favours, on the whole, assortative mating, even though the best pollen-style combinations are cross combinations.

Similar results have been obtained in pearl millet; gametophytic competition between five different genetic stocks revealed that auto-pollen had a better competitive ability than various types of allo-pollen (Sarr et al., 1988). Moreover, intergametophytic competition between pollen from wild and cultivated plants on stigmas of both wild and cultivated plants, produced preferentially wild-wild and cultivatedcultivated gamete associations (Robert et al., 1991).

Our results, indicating an opposite advantage for PGG and PTGR in self and in cross combination, suggest a buffering effect of the components of the system controlling pollen competitive ability, and could explain the lack of isolation due to this tendency in maize populations. In this experiment the segregating alleles from only two genomes were analysed (those of the parents of the RI population); however, the data suggest that the specific adaptability of the pollen for the stylar tissues of the plant for which it was selected during inbreeding favours PGG on the female of similar genotype; on the other hand it appears that physiological complementation between pollen and style of different genotypes favours PTGR on genetically divergent stylar tissues.

RFLP analysis of PGG and of PTGR on the two stylar genotypes identified nine and eight QTLs for PGG on the self and on the cross female, respectively, whereas four and 11 were detected in the case of PTGR. The corresponding coefficients of determination, high for PGG on the self and for PTGR on the cross female, low for PGG on the cross and for PTGR on the self female, indicated that more QTLs involved in the first stages of pollen function are expressed on the self female whereas factors controlling later tube growth are revealed on the cross female. Moreover, when considering the data, the statistical procedure adopted here for the detection of QTLs should be taken into account: because it is very conservative, it is possible that the number of QTLs has been underestimated.

Most of the PGCA effects map in the regions where QTLs for PGG or PTGR were detected, especially on the cross female. Some loci for PSCA effects were localized in regions already identified, but most of those concerning PTGR proved to be localized in different, not previously detected, chromosomal regions. The parameter estimated as pollen GCA is the mean tube growth rate of one pollen type in different stylar environments and measures the mean effect of the male genotype; factors conferring general adaptive features appear to be expressed when pollen germinates and grows in a genetically divergent environment. QTLs controlling specific pollen-style interaction effects were detected by means of the association between molecular markers and PSCA, the deviation of that particular pollen-style combination from the mean performance of the male genotype and from the mean performance of all the genotypes on that female plant genotype. Our data allowed specific genetic factors controlling pollen-pistil interaction to be identified, which do not coincide with genes involved in the control of pollen competitive ability per $s e$ and which, therefore, could not be revealed by simple analysis of the trait in different stylar environments.

This methodological approach can furnish useful information for plant breeding programmes, especially for the goals of the selection of genotypes in which most of the positive alleles are fixed, and of the production of parental lines maximizing combining ability (A. Camussi, personal communication).

In a previous experiment (Sari-Gorla et al., 1992), using the same RI population, the QTLs controlling pollen competitive ability were studied on the same A362 $\times$ Mo17 hybrid (cross) female. Nearly all the chromosomal regions carrying QTLs for PGG or PTGR were the same in both experiments, indicating 
an overall stability of the characters in terms of environmental and random effects; this was expected on the basis of the high values of heritability revealed by both traits: $h^{2}=0.77$ for PGG and $h^{2}=0.71$ for PTGR (Sari-Gorla et al., 1992). However, some RFLP loci which did not reveal a significant association with pollen traits in the first experiment, did in the second (for instance those on chromosome 1), and vice versa; this can be interpreted as resulting from genotypeenvironment effects, which appear to be capable of modulating the degree of gene effects.

The genes directly involved in the control of maize pollen-style interaction so far identified can be classified into different categories. Pollen bearing the recessive allele at a Gametophytic factor locus $(\mathrm{ga})$ is at a great disadvantage when competing with pollen carrying the dominant allele $(G a)$ on stylar tissues where the same dominant allele is present, but not on recessive homozygous gaga styles (Nelson, 1952, 1994). The reduced seed set (RSS) phenomenon (Rashid \& Peterson, 1992) is based on a three-gene recessive system: when the female parent is homozygous recessive for one gene (ciflocus), and when the male parent is homozygous recessive for two different genes ( $\mathrm{ciml} 1$ and cim2 loci) a reduced seed set is produced whereas the reciprocal cross is compatible. However, the precise map position of both Gametophytic factors and RSS genes is still uncertain, because of the difficulty of finding genetic markers closely linked to these genes. Many of the regions where $G a$ factors have been tentatively mapped correspond to those where many QTLs for the different components of pollen competitive ability appear to be located. In the absence of alleles with major effects, many QTLs with minor effects, such as the ones we have identified, can modulate the results of pollen competition.

\section{Acknowledgements}

The authors are grateful to T. Calinski for suggestions and discussion concerning statistical methodology, to P. Krajewski for contributing to the analysis of the data and to B. Burr for providing the recombinant inbred lines and information about their RFLP typing. The research was supported by the National Research Council of Italy, Special Project RAISA, Subproject 2, Paper number 1592.

\section{References}

BREWBAKER, J. L. AND KWACK, B. H. 1963. The essential role of calcium ion in pollen germination and tube growth. Am.J. Bot., 48, 457-464.

BURR, B., BURR, F. A., THOMPSON, K. H., ALBERTSON, C. M. AND
STUBER, c. w. 1988. Gene mapping with recombinant inbreds in maize. Genetics, 118, 519-526.

CLARKE, A. E. AND NEWBIGIN, E. 1993. Molecular aspects of selfincompatibility in flowering plants. Ann. Rev. Genet., 27, 257-279.

DICKINSON, H. 1993. Pollen dressed for success. Nature, 364, 573-574.

DUDLEY, J. W. 1993. Molecular markers in plant improvement: manipulation of genes affecting quantitative traits. Crop Sci., 33, 660 668.

FALCONER, D. S. 1989. Introduction to Quantitative Genetics, 3rd edn. John Wiley, New York.

GANESHAIAH, K. N. AND UMA SHAANKER, R. 1988. Regulation of seed number and female incitation of mate competition by a pH-dependent proteinaceous inhibitor of pollen grain germination in Leucaena leucocephala. Oecologia, 75, 110-113.

GAWEL, J. N. AND ROBACKER, C. D. 1986. Effect of pollen-style interaction on the pollen tube growth of Gossypium hirsutum. Theor. Appl. Genet., 72, 84-87.

HERRERO, M. AND ARBELOA, A. 1989. Influence of the pistil on pollen tube kinetics in peach (Prunus persica). Am. J. Bot., 76, 1441-1447.

HESLOP-HARRISON, J. 1987. Pollen germination and pollen-tube growth. Int. Rev. Cytol., 107, 1-73.

JENNINGS, D. L. AND TOPHAM, P. B. 1971. Some consequences of raspberry pollen dilution for its germination and for fruit development. New Phytol., 70, 371-380.

NELSON, O. E. 1952. Nonreciprocal cross sterility in maize. Genetics, 37, 101-124.

NELSON, O. E. 1994. The Gametophyte Factors of Maize. In: Freeling, M. and Walbot, V. (eds) The Maize Handbook, pp. 496-502. Springer, New York.

otTaviano, E. AND MUlCAHy, D. L. 1989. Genetics of Angiosperm pollen. Adv. Genet., 26, 1-64.

OTTAVIANO, E., SARI-GORLA, M. AND ARENARI, I. 1983. Male gametophyte competitive ability in maize. Selection and implications with regard to the breeding system. In: Mulcahy, D. L. and Ottaviano, E. (eds) Pollen: Biology and Implications for Plant Breeding, pp. 367-373. Elsevier, New York.

OtTaViano, E., SARI-Gorla, M. AND MUlcahy, D. L. 1980. Pollen tube growth rates in Zea mays: implications for genetic improvement of crops. Science, 210, 437-438.

OTTAVIANO, E., SARI-GORLA, M. AND VILL.A, M. 1988. Pollen competitive ability in maize: within population variability and response to selection. Theor. Appl. Genet., 76, 601-608.

PEDERSEN, s. 1988. Pollen competition in barley. Hereditas, $109,75-81$.

PFAHLER, P. L. 1967. Fertilization ability of maize pollen grains. II. Pollen genotype, female sporophyte and pollen storage interaction. Genetics, 57, 513-521.

RASHID, A. AND PETERSON, P. A. 1992. The RSS system of unidirectional cross-incompatibility in maize. I. Genetics. $J$. Hered., 83, 130-134.

ROBERT, T., LESPINASSE, R., PERNES, J. AND SARr, A. 1991. Gametophytic competition as influencing gene flow between wild and cultivated forms of pearl millet (Pennisetum typhoides). Genome, 34, 195-200. 
SARI-GORLA, M. AND FROVA, C. 1994. Pollen tube growth. In: Sahwney, V. K. and Shivanna, K. R. (eds) Pollen Biotechnology for Crops Production and Improvement. Cambridge University Press, New York (in press).

SARI-GORLA, M., OTTAVIANO, E. AND BELLINTANI, R. 1976. Competitive ability of maize pollen. Interaction between genotypes of pollen and stylar tissues. Maydica, 21, $77-80$.
SARI-GORLA, M., PÉ, M. E., MULCAHY, D. L. AND OTTAVIANO, E. 1992. Genetic dissection of pollen competitive ability in maize. Heredity, 69, 423-430.

SARR, A., SANDMEIER, M. AND PERNES, J. 1988. Gametophytic competition in pearl millet, Pennisetum typhoides (Stapf and Hubb). Genome, 30, 924-928.

SAS INSTITUTE. 1985. SAS User's Guide: Statistics. Cary, NC. 